\title{
Layered water Cherenkov detector for the study of ultra high energy cosmic rays
}

\author{
Antoine Letessier-Selvon ${ }^{\mathrm{a}, *}$, Pierre Billoir ${ }^{\mathrm{a}}$, Miguel Blanco ${ }^{\mathrm{a}}$, \\ Ioana C. Mariş ${ }^{\mathrm{a}, \mathrm{b}}$, Mariangela Settimo ${ }^{\mathrm{a}}$ \\ ${ }^{a}$ LPNHE, UPMC University Paris 6, UPD University Paris 7, CNRS/IN2P3, 4 place \\ Jussieu, FR-75252 Paris, France. \\ ${ }^{b}$ University of Granada and C.A.F.P.E., Cuesta del Hospicio, 18071, Granada, Spain
}

\begin{abstract}
We present a new design for the water Cherenkov detectors that are in use in various cosmic ray observatories. This novel design can provide a significant improvement in the independent measurement of the muonic and electromagnetic component of extensive air showers. From such multi-component data an event by event classification of the primary cosmic ray mass becomes possible. According to popular hadronic interaction models, such as EPOS-LHC or QGSJetII-04, the discriminating power between iron and hydrogen primaries reaches Fisher values of $\sim 2$ or above for energies in excess of $10^{19} \mathrm{eV}$ with a detector array layout similar to that of the Pierre Auger Observatory.
\end{abstract}

Keywords: UHECR, Mass composition, Surface array, Cherenkov detectors

\section{Introduction}

At the highest energies, above $10^{19} \mathrm{eV}$ (10 EeV or 1.6 Joules), cosmic rays are scarce (less than one per $\mathrm{km}^{2}$ per year) and with limited data on their nature. This situation dramatically limits the contribution of Ultra High Energy Cosmic Ray (UHECR) physics to fundamental physics. However, if one could access the nature of the primary particle and the details of the cascade evolution and content, substantial information would be collected about hadronic interactions above $100 \mathrm{TeV}$ center-of-mass and about the sources of such energetic particles. Aiming at an excellent primary cosmic ray identification, multiparametric measurements of Extensive Air Showers (EAS) have the potential of measuring hadronic cross sections above $100 \mathrm{TeV}$ and up to $450 \mathrm{TeV}$ centerof-mass, constraining interaction models, detecting or setting limits on UHE neutrino or gamma ray fluxes, identifying UHECR sources, and constraining Galactic and intergalactic magnetic fields.

\footnotetext{
* Corresponding author

Email address: antoine.letessier-selvon@in2p3.fr (Antoine Letessier-Selvon)
} 
Such a collection of data regarding particle interactions and cascade development at the highest energies will certainly play an invaluable role in our understanding of fundamental physics (and regarding the nature and content of our Universe). However, the limitations of current measurements regarding, in particular, the nature (or composition) of the cosmic ray flux above $50 \mathrm{EeV}$ strongly limit the coherent understanding of the available data and our capacity to address fundamental questions regarding particle interactions and transport at the highest energies, together with the nature of the sources of the particles. Even today, the existence of the Greisen-Zatsepin-Kuz'min (GZK) cut-off [1, 2] is still not totally resolved as the observed reduction in the cosmic-rays spectrum can still be interpreted as the sources "running out of steam".

From the experimental point of view it is clear that large statistics and highprecision composition measurements at the highest energy (above a few tens of $\mathrm{EeV}$ ) are needed. The current results [3 7] show that these measurements can hardly be made, with the required statistics, using current setups, in particular, because of the low duty cycle of current fluorescence detectors 8 -10].

In this paper, we introduce a novel design of water Cherenkov detectors which allows one to measure independently the muonic and electromagnetic components of the EAS and will not suffer of the duty cycle limitations of the fluorescence detection technique. The design concept and its application to water Cherenkov stations as in the Pierre Auger Observatory (Auger) are described in section 2. Its properties and performance for mass composition studies are discussed in section 3 . A first prototype has been successfully installed at the Auger site and first results are shown in section 5.

\section{Design principles}

\subsection{Why count muons}

After the few first interactions, EAS generated by UHECR contain a very large number of particles undergoing an even larger number of interactions. One can therefore expect that the cascade description can be modeled from a reduced set of universal functions that will depend on the shower age and whose relative amplitudes will carry the information on the primary type and energy. This "universality" concept [11, 12 has been extensively studied and validated with Monte Carlo simulations and, indeed, the arrival direction, the depth of shower maximum $\left(\mathrm{X}_{\max }\right)$ and the muon size at ground $\left(\mathrm{S}_{\mu}\right)$ effectively contain the essential information about the primary UHECR. It follows that one can parametrize the ground signal of EAS with a set of universal functions (predicted or measured and only dependent on those macroscopic quantities), from which the relevant information about the primary UHECR can be retrieved [12.

The principle of cosmic ray mass determination using the muon densities at ground and the shower maximum is illustrated in figure 1 where a set of $10 \mathrm{EeV}$ proton and iron simulated showers at 38 degrees zenith angle has been used. It is clear that, once the energy is known, a selection criterion can be built in the $\mathrm{X}_{\max } \mathrm{vs}_{\mu}$ plane that permits a very high efficiency/purity separation between 


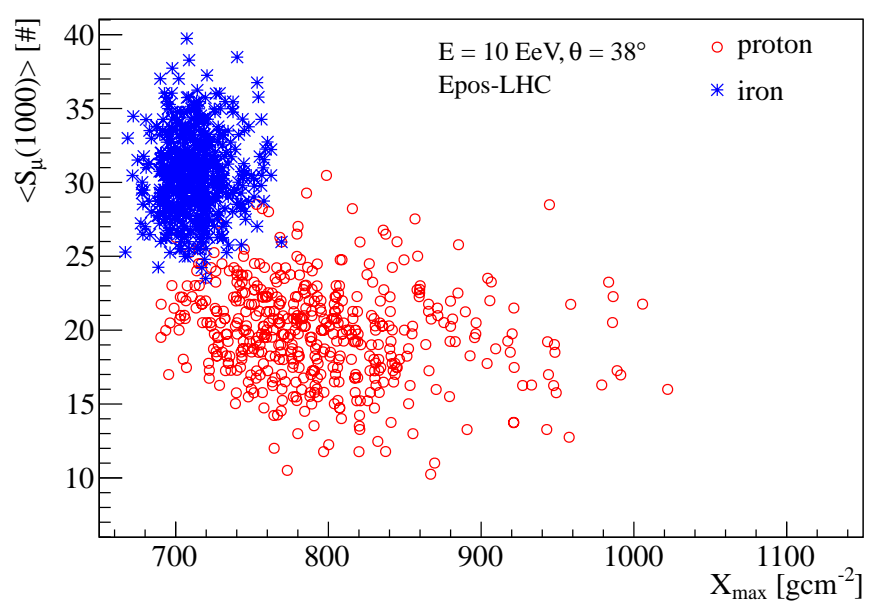

Figure 1: Average number of muons, $\left\langle\mathrm{S}_{\mu}(1000)\right\rangle$, entering a $10 \mathrm{~m}^{2}$ detector versus $\mathrm{X}_{\max }$ for 500 iron (blue) or proton (red) showers at $10 \mathrm{EeV}$ and $38^{\circ}$ zenith angle. The value for $\mathrm{S}_{\mu}$ corresponds to the average of four measurements. The $\mathrm{X}_{\max }$ distribution (horizontal spread) is due to the shower to shower development fluctuations for a given primary mass at fixed zenith angle and energy.

the two species. Hence, a detector design that can perform an appropriate measurement of both these quantities (or related ones) should provide the necessary information to analyse cosmic ray data within definite mass samples. In figure 1 no detector resolution is taken into account. In the next sections the impact of a more realistic situation is presented. However, it is evident from this figure that, due to shower to shower fluctuations, a resolution of about $20 \mathrm{~g} / \mathrm{cm}^{2}$ for $\mathrm{X}_{\max }$ is needed to infer the mass composition on the event by event basis. On the other hand, a resolution of about 10 to $20 \%$ for $\mathrm{S}_{\mu}$ is also suitable given Poisson fluctuations in the muon numbers at ground and the limited surface area of muon counters that can be realistically deployed $\left(\sim 10 \mathrm{~m}^{2}\right)$.

\subsection{Layered system}

Energy absorption in water Cherenkov detectors (WCD) can be used to distinguish muons from the electromagnetic (EM) component of EAS. The electromagnetic particles $\left(\mathrm{e}^{+} / \mathrm{e}^{-}\right.$and photons) reaching the ground have an average energy of about $10 \mathrm{MeV}$. Electrons and positrons are absorbed in water over a few centimeters while photons deposit their energy over a radiation length $(\sim 36 \mathrm{~cm})$. On the other hand muons, having a typical average energy of a few $\mathrm{GeV}$, can go through several meters of water without being stopped. If the water volume of a WCD is split into two horizontal layers, one of which being at least partially shielded from the electromagnetic component, then the two volumes will provide distinct responses to the EAS components. A Layered Surface Detector (LSD) can therefore provide the means to reconstruct independently, 

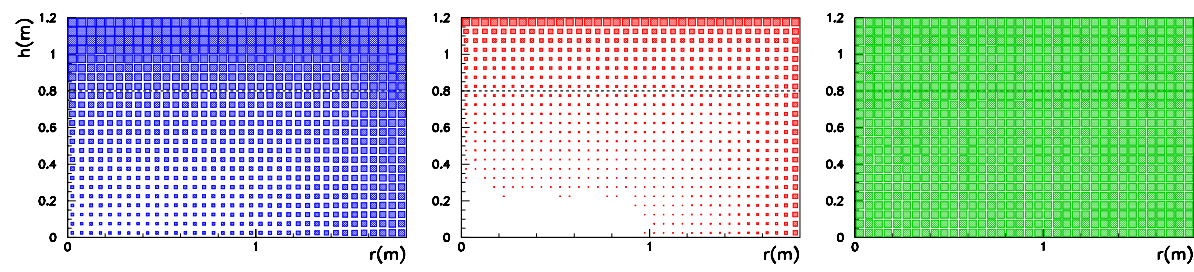

Figure 2: Distribution of the Cherenkov photons production point in a $1.2 \mathrm{~m}$ height and $1.8 \mathrm{~m}$ radius WCD. From left to right the contribution from the photons, $e^{+} e^{-}$and muon component of a $30 \mathrm{EeV}$ EAS with $45^{\circ}$ zenith angle is shown.

for each EAS, the muonic and electromagnetic components. One remarkable property is that the shielding of the muon sensitive volume does not need to be very large: in practice a single radiation length is enough to achieve good performance. This is due to the fact that WCD have a distinct response to the EM and muonic component of EAS. Indeed, whereas EM particles of the EAS are absorbed in the water volume in a calorimetric way, muons are minimum ionizing particles and deposit an amount of energy proportional to their track length (i.e., several hundreds of $\mathrm{MeV}$ for a meter depth detector).

In its simplest form, a LSD is composed of two independent and light-tight volumes that are created by inserting a horizontal reflective layer in a water volume. Other implementations with more than two layers and/or shielding on the side can also be imagined. However the simple design described here is enough to show the main properties of such a detector. In the next section, a modification of the WCD design from the Auger surface array [13] is presented based on this design.

\subsection{A LSD design from the Auger WCDs}

The Auger WCD [14] have a $1.8 \mathrm{~m}$ radius by $1.2 \mathrm{~m}$ height water volume, overlooked by three 9 inch photomultiplier tubes. In figure 2 we show the production point of Cherenkov photons in such detectors, for a $30 \mathrm{EeV}$ shower with $45^{\circ}$ zenith angle. As expected the EM component dominantly deposits its energy on the top and side of the station.

The two water volumes of the LSD can be created by inserting a horizontal reflective layer at a height of $80 \mathrm{~cm}$ from the WCD bottom 1 . This is represented as a dashed line in figure 2. The three PMTs that equip the Auger WCD are left in place. In the upper layer, the EM component of an EAS will deposit most of its energy, while the $80 \mathrm{~cm}$ bottom layer will detect the much more penetrating muons (and to a lesser degree the surviving EM particles).

A central cylinder of about 10 inch diameter provides the mechanical structure as well as the enclosure for the additional 9 inch photo-tube that collects

\footnotetext{
${ }^{1}$ This choice is justified in the next paragraph
} 

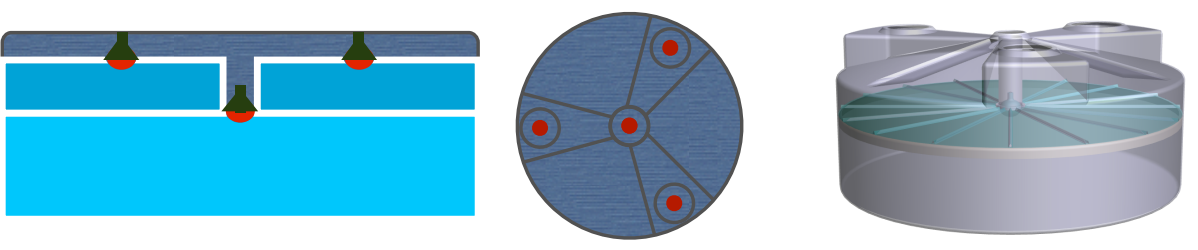

Figure 3: Schematic and artistic view of a LSD build from an Auger WCD design.

the light from the bottom layer. Some simplified schematics and an artistic view of this LSD is shown in figure 3 .

\subsection{Signal extraction}

The reconstruction of the EM and muonic component of EAS relies on the fraction of the signal deposited by each component in each of the two layers. These fractions define a $2 \times 2$ matrix $\mathcal{M}$ that gives the measured top and bottom signal ( $S_{\text {top }}$ and $S_{\text {bot }}$ respectively) as a linear superposition of the EM $\left(S_{\mathrm{EM}}\right)$ and muon $\left(S_{\mu}\right)$ contributions (the column sums of the matrix are one by construction),

$$
\left(\begin{array}{c}
S_{\mathrm{top}} \\
S_{\mathrm{bot}}
\end{array}\right)=\mathcal{M}\left(\begin{array}{c}
S_{\mathrm{EM}} \\
S_{\mu}
\end{array}\right)=\left(\begin{array}{cc}
a & b \\
1-a & 1-b
\end{array}\right)\left(\begin{array}{c}
S_{\mathrm{EM}} \\
S_{\mu}
\end{array}\right)
$$

Hence, if the matrix $\mathcal{M}$ can be inverted, the muonic and EM signal deposition in the LSD can be retrieved as :

$$
\left(\begin{array}{c}
S_{\mathrm{EM}} \\
S_{\mu}
\end{array}\right)=\mathcal{M}^{-1}\left(\begin{array}{c}
S_{\mathrm{top}} \\
S_{\mathrm{bot}}
\end{array}\right)
$$

The determinant $\mathcal{D}$ of the $\mathcal{M}$ matrix is $a-b$ and maximises when $\mathcal{M}$ is equal to the identity $(a=1$ and $b=0)$. In a realistic situation $a$ is always less than one while $b$ is always larger than zero, hence $|\mathcal{D}|$ will be less than one. This is important as the statistical uncertainty in the reconstructed muonic and EM signals from measurements in the top and bottom layer are driven by $1 / \mathcal{D}$, the determinant of $\mathcal{M}^{-1}$.

The coefficients $a$ and $b$ depend on the geometry of the two water volumes and on the efficiency of the light collection. They can be obtained from well established simulations of the tank response. A rough estimate of $a$ and $b$ can be derived for a vertical incidence, when neglecting the signal produced by particles entering through the side of the detector. In fact, modelling the absorption of the electromagnetic component by an exponential decay according to the radiation length $X_{0}$ and for a tank of height $H$ with a layer interface located at a distance $H-h$ from the bottom we have :

$$
a=1-e^{-h / X_{0}} \text { and } b=\frac{h}{H} \text { with } h \in[0, H]
$$



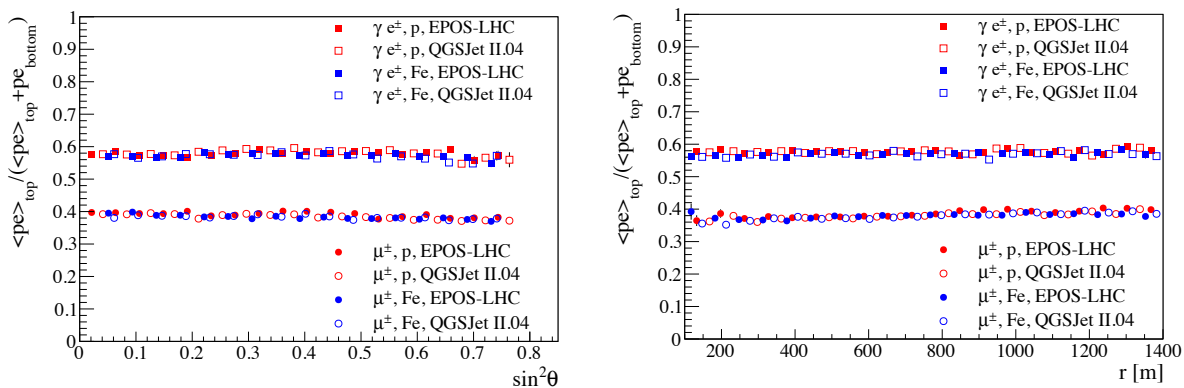

Figure 4: Fraction of photo-electrons (pe) collected in the top layer for the EM (square symbols, $a$ coefficient of the matrix) and muonic (round symbols, $b$ coefficient) component for two hadronic models. Left : dependence as a function of zenith angle. Right: dependence as a function of distance to the shower core. The coefficients $a$ and $b$ are essentially independent of the shower characteristics or detector distance from the shower core, they only depend on the tank geometry.

$\mathcal{D}=a-b$ is maximum for $h=X_{0} \ln \left(H / X_{0}\right)$. If $H$ is large enough (keeping the radius also large so that the side contributions can still be neglected) $a$ goes to 1 while $b$ goes to 0 and $\mathcal{M}$ tends towards the ideal unity matrix. In a more realistic case, particles entering from the side wall of the station cannot be neglected and the optimal values of $a$ and $b$ also depend on the proportion between the height and the radius of the tank.

In the particular case of the Auger WCD, $H$ is $120 \mathrm{~cm}, X_{0}=36 \mathrm{~cm}$ giving $h=43 \mathrm{~cm}$. An optimal position for the interface layer is therefore at about $80 \mathrm{~cm}$ from the bottom of the water tank.

\section{Performances}

\subsection{Matrix universality}

To precisely calculate $a$ and $b$ and to characterize the performances of the LSD, simulations of the detector response have been performed. Air showers have been simulated with the CORSIKA code [15], using EPOS-LHC [16] and QGSJetII.04 [17] as high energy interaction models and FLUKA [18] at low energy. Various libraries have been generated with a uniform distribution in $\cos ^{2} \theta$ for different primary type (proton, helium, nitrogen and iron) and in two energy intervals (from 8 to $13 \mathrm{EeV}$ and from 40 to $60 \mathrm{EeV}$, uniformly distributed in the logarithm of energy).

The matrix coefficients $a$ and $b$ are derived from simulations as the ratio between the photo-electrons collected in the top layer and the total number of photo-electrons in the two volumes for the EM and muonic components respectively. A remarkable property of the LSD is that the coefficients $a$ and $b$ are essentially independent of the UHECR primary type and energy and also of the particular simulation model used to describe the EAS. This is shown in figure 4 

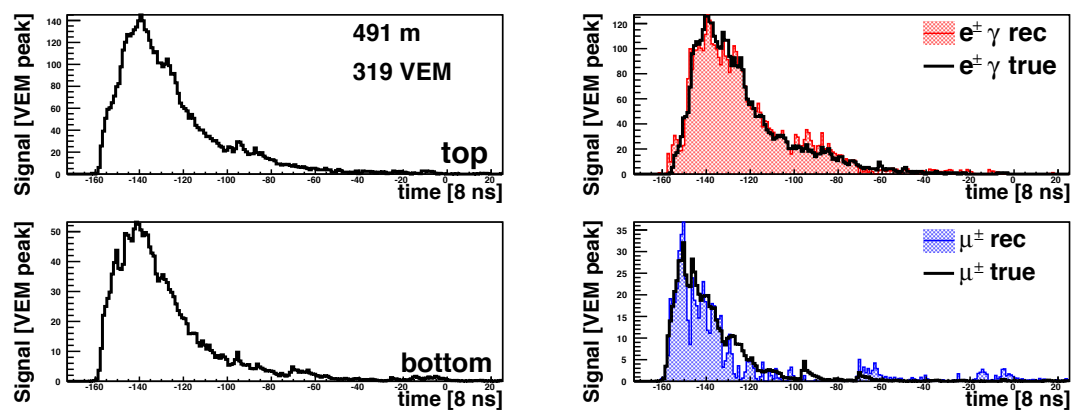

Figure 5: Simulation of the signal collected in the top (bottom) part of a $10 \mathrm{~m}^{2}$ LSD for a 11 $\mathrm{EeV}$ shower with $46^{\circ}$ zenith angle and 491 meters away from the core. The $\mathcal{M}$ reconstruction of the muon and EM traces compared to the generated ones are shown in the right panel. The agreement is striking both in shape (timing information of the two components) and amplitude. This hints at the excellent performances that can be expected from this design for the multi-component study of EAS.

where the coefficient $a$ and $b$ have been evaluated for different primaries and hadronic models and are shown as a function of zenith angle and distance to core. It is notable that for the Auger WCD geometry, $a$ and $b$ are also essentially independent of the shower zenith angle in the range $\left[0,60^{\circ}\right]$. This is due to a compensation between the top and side wall contributions coming from the particular geometry of the Auger WCD which have an height over radius ratio of $2 / 3$.

For the particular case of the LSD from the modified Auger WCD the parameter $a$ is nearly 0.6 while $b$ is about 0.4 (see figure 4), leading to a determinant $\mathcal{D}=1 / 5$.

\subsection{Signal reconstruction}

For ground arrays the reconstruction of the primary UHECR properties relies on the adjustment of a lateral distribution function (LDF) that describes the detector signals as a function of their distance from the core. The value of the LDF at a reference distance to cor $\AA^{2}$ serves as an energy estimator but other characteristics such as its slope also reflect EAS properties, for instance its age. Using the LSD, it is possible to derive two independent LDFs, one for the muonic and one for EM component, by means of the corresponding signals reconstructed in each station. This offers the additional possibility to perform an energy reconstruction that takes into account the muon size and shower age and to produce a calibration based only on the electromagnetic component. Such a procedure would be less sensitive to the shower to shower fluctuation

${ }^{2}$ The reference distance is chosen as the one which minimizes the fluctuations of the expected signal, due to the lack of knowledge on the LDF. It is a function of the array grid spacing and the EAS energy range studied. It is $1000 \mathrm{~m}$ for Auger [19]. 

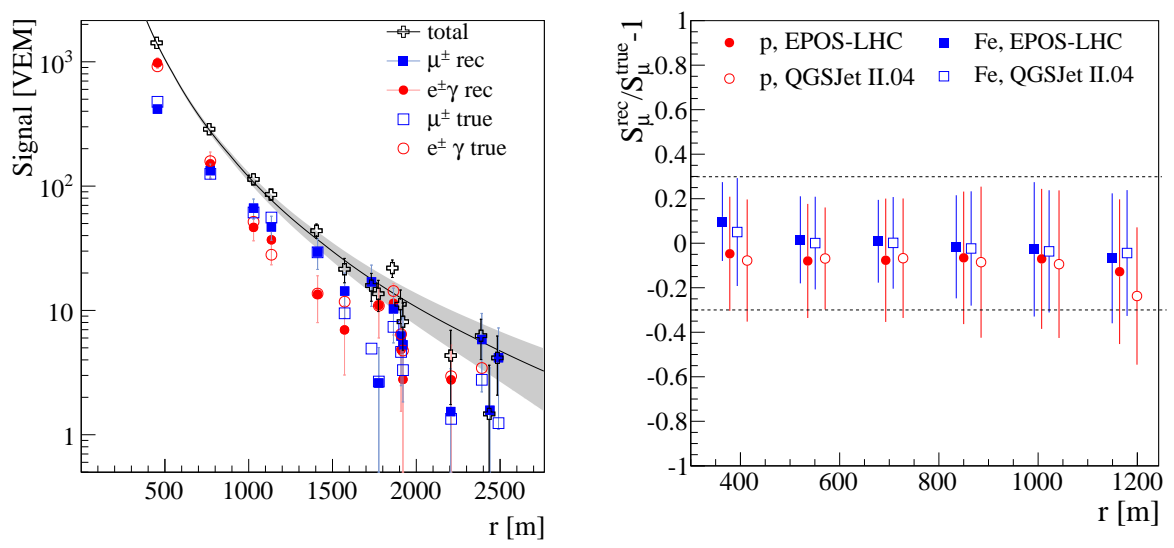

Figure 6: Left: example of muonic and EM independent LDFs reconstructed using the individual signal reconstruction in each of the detectors. Right: individual LSD muon signal reconstruction as a function of the distance to core, the error bars represent the signal resolution.

and to the interaction models than the one that uses the mixed EM and muonic signals.

Inverting the matrix $\mathcal{M}$, it is possible to construct for each detector FADC traces for the two components separately. An example is shown in figure 5 for an $11 \mathrm{EeV}$ shower at $46^{\circ}$ zenith angle. This graph alone demonstrates the power of the LSD to accurately determine the muonic and electromagnetic components of the EAS, for both the integrated signals and their time distribution. The muonic and electromagnetic LDFs can easily be reconstructed from these individual measurements, as shown in figure 6 (left).

\subsection{Mass separation}

With the LSD one can reconstruct the muon signals from at distances to core of nearly $200 \mathrm{~m}$ to more than $2 \mathrm{~km}$ for the highest energetic showers. As shown in figure 6 (right), the signal resolution in each detector is better than $25 \%$ when more than 20 muons enter the detector while the Poisson fluctuations dominate for smaller signals. These resolutions are given for $10 \mathrm{EeV}$ and they improve significantly above $40 \mathrm{EeV}$, e.g. $<14 \%$ for the muon size.

By following the usual approach for the shower size reconstruction with surface array detectors, a measurement of the muon size of EAS can be obtained from the muonic LDF, at a reference distanc ${ }^{3}$ The global resolution on the EAS muon size parameter is $20 \%$ (16\%) for proton (iron) at $10 \mathrm{EeV}$, improving to about $10 \%$ for both at $70 \mathrm{EeV}$ (see figure 7 , left).

\footnotetext{
${ }^{3}$ We adopt here the same reference distance as in Auger $(1000 \mathrm{~m})$, but optimal values can be derived in the future.
} 

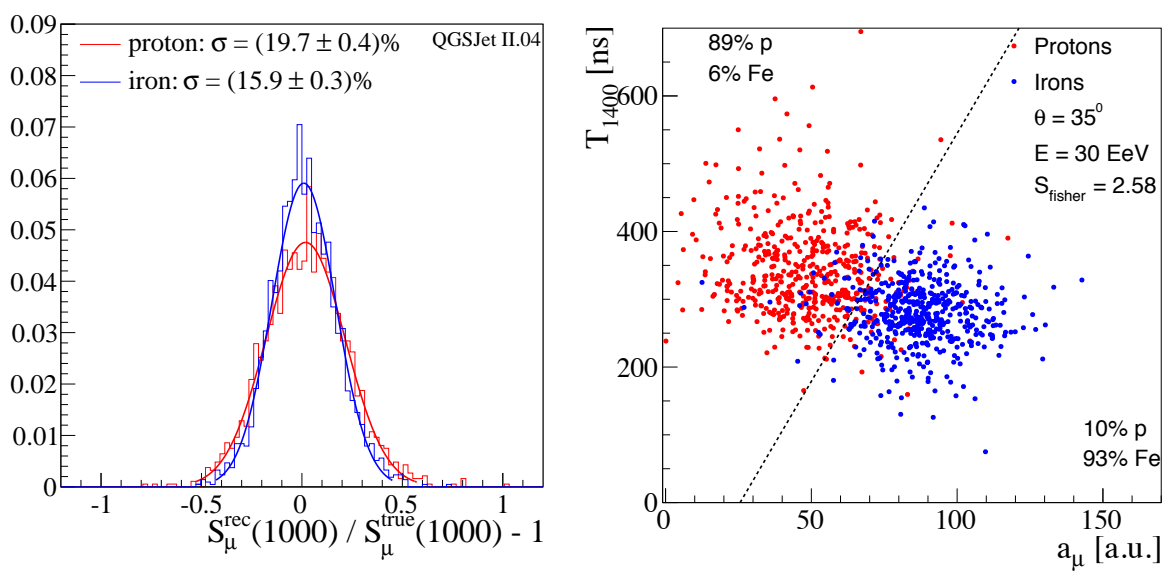

Figure 7: Left: global resolution on the muon size at $1000 \mathrm{~m} S_{\mu}$ for air showers with energy between 8 and $13 \mathrm{EeV}$. Right: Example of proton / iron separation plot at $30 \mathrm{EeV}$ from the LSD reconstructed start time and muon signal size. The horizontal axis is the muon size at $1000 \mathrm{~m}$ from the shower core in arbitrary units, it is obtained by a simple LDF fit to the reconstructed muon signal in each WCD. The vertical axis is the start time at $1400 \mathrm{~m}$ (see text).

The muon size can be combined with $\mathrm{X}_{\max }$ or with other age sensitive parameters to produce a two or multi-dimensional plot as discussed in the introduction, improving the mass composition separation capabilities. An $\mathrm{X}_{\max }$ sensitive parameter can also be retrieved following the universality principle of EAS description, as for example using the approach proposed in [12, 20]. An example of the separation power for a $50 \%$ proton $50 \%$ iron mixed composition at $30 \mathrm{EeV}$ is given in figure 7 (right). The muon signal reconstructed with the LSD is plotted against the signal start time at $1400 \mathrm{~m}\left(\mathrm{~T}_{1400}\right)$ which measures the delay of the shower particles with respect to the arrival time of a imaginary planar front. This parameter is sensitive to the shower front curvature and correlates to $\mathrm{X}_{\max }$. In this particular example we have used a timing resolution of $8 \mathrm{~ns}$ which can be easily achieved with modern GPS receivers. This correspond to an $\mathrm{X}_{\max }$ resolution of 40 to $60 \mathrm{~g} / \mathrm{cm}^{2}$ depending on zenith angle. The Fisher separation coefficient for this particular case is larger than 2 indicating that excellent separation power. It is worthwhile noting that this merit factor is obtained for a fixed energy in the simulation. In a realistic scenario both, the energy and $\mathrm{S}_{\mu}$, need to be estimated from the LSD data. It is to be expected that this will diminish the proton-iron separation, but since we can experimentally estimate the energy by combining the $\mathrm{X}_{\max }$ (or $\mathrm{T}_{1400}$ ) with the shower size of the electromagnetic component at ground, a merit factor of better than 2 will be possible. The parameter $T_{1400}$ is given as example and it is not meant to be considered as the optimal variable for the analysis. A detailed study of $\mathrm{X}_{\max }$ (or age) sensitive parameters and of the mass separation is in progress and is however out of the scope of this paper. 

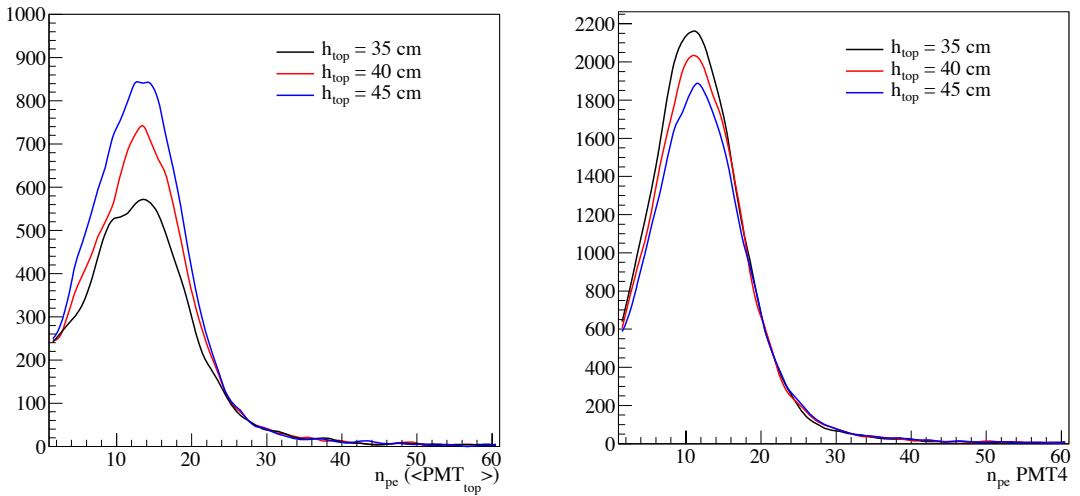

Figure 8: Signals from the electrons of muon decaying in the LSD tanks. Left: average signal in the three top PMTs. Right: signal in the bottom PMT. The statistics in the plot corresponds to about a 15 minute run or 50,000 decays.

\section{Calibration strategy}

An important aspect of surface array detectors for UHECR studies is to have a calibration strategy that allows to monitor the conversion of the electronic signals into an equivalent energy deposit (or particle count).

\subsection{Muon peak}

In the Auger surface array, the calibration of the WCD is based on the energy deposited by a vertical muon traversing the WCD volume at its centre [21, 22]. The energy deposited by such muons is about $240 \mathrm{MeV}$ and is called a VEM (Vertical Equivalent Muon). The constant flux of atmospheric muons (about 3000 go through the Auger WCD each second) provides such a calibration. Indeed, the distribution of the charge collected at the dynode of any of the 3 PMT of the Auger WCD shows a hump (see figure 9) whose maximum corresponds nearly to one VEM of energy deposited in the tank. The exact correspondence between the VEM and the most probable charge collection has been measured in Auger using a scintillator telescope, and it is constant in time with only a few percent variation over several years. Such a procedure gives the absolute calibration of individual PMTs.

For the absolute PMT calibration in the LSD a technique based on the VEM value, similar to the one currently used in Auger, can be adopted. An example for the bottom segment charge histogram of our prototype (see next section for a detailed description) is shown in figure 9 . From the ADC counts corresponding to the maximum of the VEM charge histogram, one can find the correspondence between ADC counts and the amount of energy deposited in the water volume.

\subsection{Muon decay}

An alternative calibration can be obtained using the muon decays that occur in the water volume. Nearly $4 \%$ of the muons entering the WCD stop and decay 
and the Michel electrons deposit on average an amount of energy that can also be used for calibration purposes.

For the LSD it is also convenient to determine the water volumes geometry, i.e. the precise height of the water separation interface $(h)$ as the matrix coefficients $a$ and $b$ depend on it. The muon decay rate in the top and bottom segments is determined by the geometry of the station and hence allows for a precise determination of the segment position $h$.

In figure 8 we show a simulation based on 50,000 muon decays (this can be obtained in about 15 minutes assuming a decay selection efficiency of 50\%) for three different positions of the interface. A $5 \mathrm{~cm}$ difference corresponds to nearly 10 standard deviations.

A precision of a few millimeters can thus be achieved.

\subsection{Hybrids and physics data}

A cross calibration of the matrix coefficient within an array of LSD is also possible based on physics results. Average (top and bottom) LDFs from a set of events can be constructed (an example is given in figure 10) for each individual LSD. From these average LDFs and using the matrix one can reconstruct independently for each LSD average muonic and EM LDFs. Since these average LDFs should be identical for all LSDs this is a means to cross calibrate all matrices.

Finally in a hybrid observatory such as Auger 13 or the Telescope Array [23, the EM LDFs from the LSD can be calibrated using the fluorescence telescope data that give the cosmic ray energy by means of a calorimetric measurement of the EM energy deposit in the atmosphere [24, 25]. This calibration scheme in the Auger Observatory uses the total signal in the WCDs. In the LSD it will benefit from the EM signal reconstruction as the uncertainty on the muon number in individual showers will no longer deteriorate the energy resolutions of the surface array.

\subsection{Propagation of uncertainties and systematics}

When reconstructing the LDF from individual signals $(S)$ measured in standard WCDs the uncertainty of each measurements is of the order of $\sqrt{S}$ with $S$ expressed in VEM units. This is due to the Poisson fluctuation of the number of muons entering the tank and to the fluctuation of the EM component in its high energy tail which also introduce VEM size Poisson fluctuations. The additional uncertainty associated with the signal measurement in the tank itself is due to the photo-electron (p.e.) statistics. As long as we have number of p.e. per VEM much larger than one, this contribution to the error budget is negligible.

Above $10 \mathrm{EeV}$, the signal at $1000 \mathrm{~m}$ is at least several tens of VEMs and the particle fluctuations due to the detector sampling become negligible compared to the shower to shower fluctuations. ndeed above $10 \mathrm{EeV}$ the energy resolution obtained from the LDF size at $1000 \mathrm{~m}$ from the Auger WCD is $12 \%$ [26] while one would naively expect around 5 to $6 \%$ if the particle count was alone responsible for this uncertainty. 

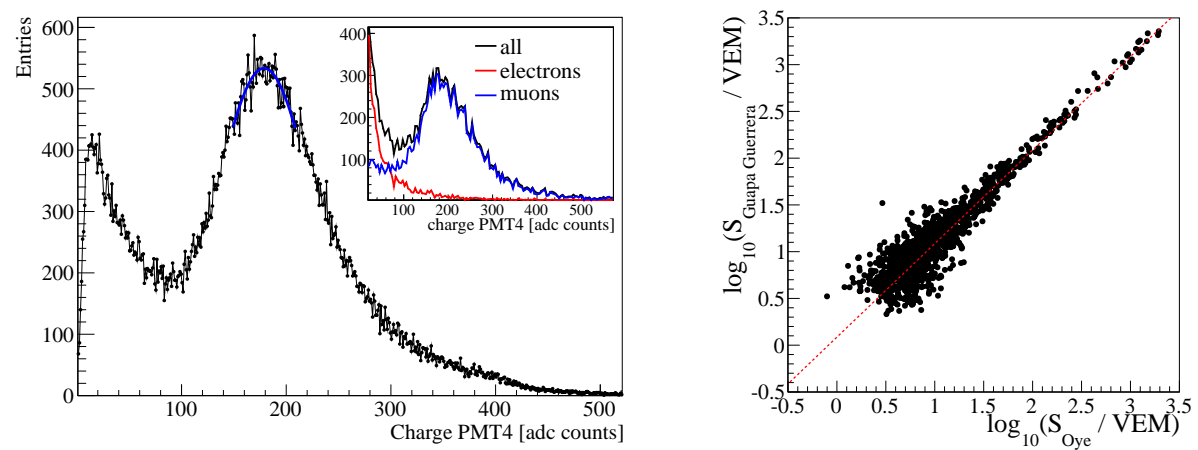

Figure 9: Left: muon peak from the charge distribution of the bottom PMT of our prototype data compared with a GEANT4 simulations of a LSD (insert). Right: total signal in our LSD prototype (Guapa Guerrera) compared to a standard WCD (named Oye) of the Auger array located $10 \mathrm{~m}$ away. As expected, there is a linear relation between the sum of the signals in the two LSD segments and a standard tank.

In an LSD tank the situation is similar even though the reconstructed muon and EM signals are linear combinations of the top and bottom signals with rather large coefficients. For a matrix with coefficients $a=0.6$ and $b=0.4$ we have :

$$
S_{E M}=3 S_{t o p}-2 S_{b o t} \text { and } S_{\mu}=3 S_{b o t}-2 S_{t o p}
$$

However the particle fluctuations in the top and bottom segment are correlated while the p.e. count fluctuations are not. In the particular case of the LSD geometry considered in this article, the contribution of the p.e. count fluctuations in the reconstructed $S_{\mu}$ and $S_{E M}$ signals in each tank, although amplified by the large coefficients of equation 4 is still less than $25 \%$ of the particle fluctuations themselves. This has little impact on the total error budget, especially at the highest energies where shower to shower fluctuations dominate.

Due to the relatively large coefficients entering in the reconstruction of $S_{\mu}$ and $S_{E M}$ one must also consider the effect of a possible systematic in the absolute calibration of the top and bottom segments of the LSD. As an illustration, assume that the calibration procedure induces a systematic bias of $+1 \%$ on the top segment with respect to the bottom one over the whole array. In such case the EM signal from equation 4 will be on average 3\% too large and the muon signal $2 \%$ too small. Still, the LSD system can separate heavy from light primaries according to the muonic content of the EAS as all primaries will suffer the same systematic shift in the energy vs muon size plane. The comparison with models becomes more difficult since the electromagnetic size, hence the energy, will be overestimated while the muon size will be underestimated. Given the calibration strategy depicted above a maximum of $1 \%$ systematic between the top and bottom calibration is within reach but requires attention. 

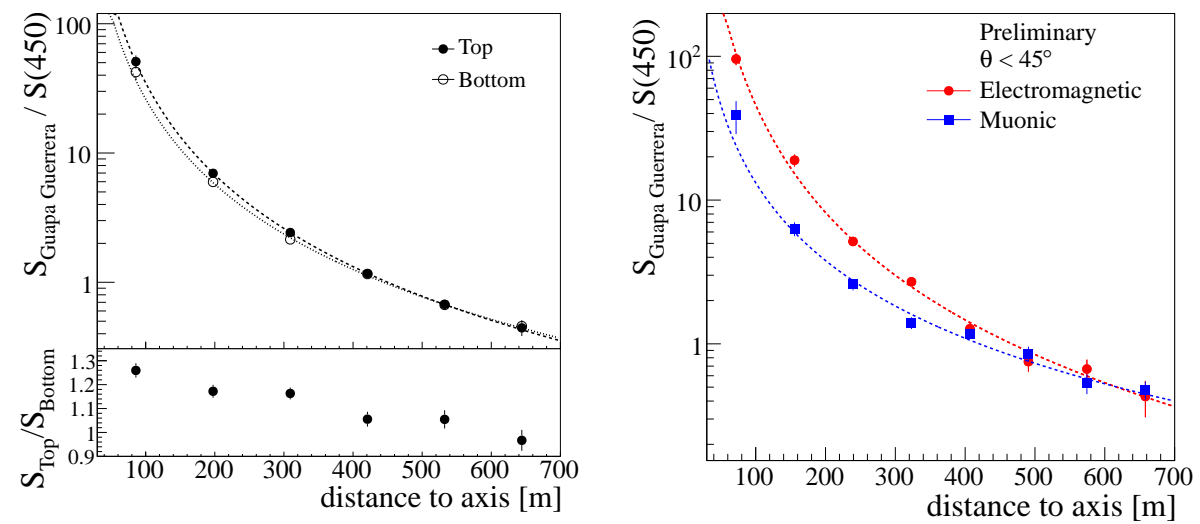

Figure 10: Average LDF from the LSD prototype running at the Pierre Auger Observatory. 710 showers with reconstructed energies above $0.03 \mathrm{EeV}$, zenith angle below $45^{\circ}$ and Guapa Guerrera located at a distance of less than $700 \mathrm{~m}$ have been selected for this plot. This corresponds to two weeks of data taking. Left: LDFs reconstructed with the signal recorded in the top and bottom layers separately. Right: preliminary muon and EM LDFs reconstructed applying a matrix with $a=0.6$ and $b=0.4$ to the top and bottom signals.

\section{Prototype test}

A LSD prototype was constructed at the Auger site in Malargüe inserting a separation, made from a Tyvek laminate, in one of the Auger liners. This prototype uses the 4 PMT configuration displayed in figure 3 with 3 PMTs looking into the top volume and 1 PMT looking in the bottom one. This LSD, named "Guapa Guerrera", was installed in the field during the last week of February 2014 and has been smoothly taking data since then. We report here on the first two weeks of data collected by this prototype.

\subsection{Calibration}

The histogram of charge of random signals for the PMT observing the bottom volume, as recorded by the local acquisition system [21, is illustrated in Figure 9 , left. From this figure one clearly distinguishes the peak which corresponds to the charge deposited by single muons that is roughly one VEM (the exact correspondence will be measured with a muon telescope) and can be used for calibration.

In the same figure, right, we have compared the total signal recorded in the LSD (by summing after a preliminary calibration of top and bottom signals) to the signal recorded in the same events by a standard Auger WCD located $10 \mathrm{~m}$ away. We found, as expected, a linear correlation between the two signals over nearly three orders of magnitude. This shows that the LSD can also be used and can perform like a standard WCD. 


\subsection{Muonic and electromagnetic $L D F$ reconstructions}

The LSD prototype is located in the infill part of the Auger surface array, a region with a WCD density 4 times higher than in the regular array [27. It participates in about 100 physics events per day. In the first two weeks of operation we recorded about 1400 events out of which we selected 710 events with a reconstructed energy above $0.03 \mathrm{EeV}$ and a zenith angle less than $45^{\circ}$. We have also requested that the energy deposit in the top part of the detector is larger than $\approx 400 \mathrm{MeV}$. The zenith angle cut ensures that, even for those relatively low energy showers that develop higher in the atmosphere, the EM component is not completely absorbed before reaching ground. After normalizing the individual LDF of each events at $450 \mathrm{~m}$ it is possible to plot, as a function of distance to cores, the signal recorded by the Guapa Guerrera prototype in all of those events. This "average LDF" is shown on the left panel of figure 10 for the top and bottom segments separately. On can see from this plot (and the accompanying lower panel showing the evolution of the top to bottom signals ratio) that the top average LDF is steeper than the bottom one. This is expected since the top segment has a larger contribution from the EM component which has a steeper LDF than the muonic one. On the right panel of figure 10 we show the reconstructed average EM and muonic LDFs obtained from equation 4 applied to the top and bottom average LDFs. This result is preliminary as we used the nominal values of the matrix coefficients $(a=0.6$ and $b=0.4$, not determined for the exact geometry of Guapa Guerrera) and preliminary calibration constants. Nevertheless the quality of this result obtained after just two weeks of data taking is very promising. In addition, and as stated previously, the reconstruction of average muonic and EM LDFs using different LSD stations will allow us to cross calibrate the individual matrices based on the requirement that all LSDs are looking at the same physics.

\section{Conclusions}

We have presented the LSD (Layered Surface Detector) as a new concept of water Cherenkov tank that allows us to reconstruct mass sensitive parameters for UHECR with optimal resolution. The muon size of EAS can be reconstructed with a precision better than $20 \%$ above $10 \mathrm{EeV}$, reaching $10 \%$ for energies above $70 \mathrm{EeV}$. The separation of muonic and electromagnetic lateral distributions on an event by event basis further provides an estimation of the $\mathrm{X}_{\max }$ parameter with $50 \mathrm{~g} / \mathrm{cm}^{2}$ resolution from timing alone. It could be reduced to $30 \mathrm{~g} / \mathrm{cm}^{2}$ by including the time shape information.

A prototype of the LSD, constructed from a modification of one of Auger surface array WCD, has shown excellent performances in agreement with expectations from Monte-Carlo simulations.

We argue that such detectors should be seriously considered for any upgrade of existing UHECR observatories or for the construction of new observatories, either with larger aperture than the current one or dedicated to the study of the second knee to ankle region, that is in the energy range from $0.1 \mathrm{EeV}$ to $10 \mathrm{EeV}$. 


\section{Acknowledgments}

We gratefully acknowledge the very fruitful exchanges we had with all of our colleagues in the Auger collaboration and the use of all Auger facilities from hardware to software including access to a subset of shower data. We are also deeply indebted to the commitment of the observatory staff whose strong support in constructing and deploying the LSD prototype was extremely appreciated. The work of M.S. and I.C.M. made in the ILP LABEX (ANR10-LABX-63), is supported by French state funds managed by the ANR within the Investissements d'Avenir programme (ANR-11-IDEX-0004-02). I.C.M. also acknowledges the financial support by the European Community 7th Framework Program, through the Marie Curie Grant FP7-PEOPLE-2012-IEF, no. 328826.

\section{References}

\section{References}

[1] K. Greisen, End to the cosmic ray spectrum?, Phys. Rev. Lett. 16 (1966) 748.

[2] G. Zatsepin, V. Kuzmin, Upper limit of the spectrum of cosmic rays, JETP Lett. 4 (1966) 78.

[3] R. U. Abbasi, et al., Indications of Proton-Dominated Cosmic Ray Composition above 1.6 EeV, Phys. Rev. Lett. 104 (2010) 161101, arXiv: 0910.4184 .

[4] J. Abraham, et al., Measurement of the Depth of Maximum of Extensive Air Showers above $10^{18} \mathrm{eV}$, Phys. Rev. Lett. 104 (2010) 091101, arXiv: 1002.0699.

[5] E. Barcikowski, et al., Mass Composition Working Group Report at UHECR-2012, EPJ Web Conf. 53 (2013) 01006, arXiv:1306.4430.

[6] T. Abu-Zayyad, et al., Pierre Auger Observatory and Telescope Array: Joint Contributions to the 33rd International Cosmic Ray Conference (ICRC 2013), Proc. of 33rd Int. Cosmic Ray Conf., Rio de Janeiro, Brazil, arXiv: 1310.0647 .

[7] L. Dedenko, G. Fedorova, T. Roganova, A. Glushkov, S. Knurenko, et al., The composition of the primary particles at energies $3 \times 10^{17}-3 \times 10^{19} \mathrm{eV}$ observed at the Yakutsk array, J. Phys. G39 (2012) 095202.

[8] J. Abraham, et al., The Fluorescence Detector of the Pierre Auger Observatory, Nucl. Instrum. Meth. A620 (2010) 227, arXiv:0907.4282.

[9] P. Abreu, et al., The exposure of the hybrid detector of the Pierre Auger Observatory, Astropart. Phys. 34 (2011) 368, arXiv:1010.6162. 
[10] H. Tokuno, Y. Tameda, M. Takeda, K. Kadota, D. Ikeda, et al., New air fluorescence detectors employed in the Telescope Array experiment, Nucl. Instrum. Meth. A676 (2012) 54, arXiv:1201.0002.

[11] P. Lipari, Universality of cosmic ray shower development, Nucl. Phys. Proc. Suppl. 196 (2009) 309.

[12] M. Ave, R. Engel, J. Gonzalez, D. Heck, T. Pierog, M. Roth, Extensive air shower universality of ground particle distributions, Proc. 32nd ICRC, Beijing, 2011, vol 2, 178.

[13] J. Abraham, et al., Properties and performance of the prototype instrument for the Pierre Auger Observatory, Nucl. Instrum. Meth. A523 (2004) 50.

[14] I. Allekotte, et al., The surface detector system of the pierre auger observatory, Nucl. Instrum. Meth. A586 (2008) 409, arXiv:0712.2832.

[15] D. Heck, J. Knapp, J. Capdevielle, G. Schatz, T. Thouw, Corsika: a monte carlo code to simulate extensive air showers, Wissenschaftliche Berichte, Forschungszentrum Karlsruhe FZKA 6019.

[16] K. Werner, I. Karpenko, T. Pierog, Ridge in proton-proton scattering at 7 TeV, Phys. Rev. Lett. 106 (2011) 122004.

[17] S. Ostapchenko, Monte Carlo treatment of hadronic interactions in enhanced Pomeron scheme: I. QGSJET-II model, Phys. Rev. D83 (2011) 014018, arXiv: 1010.1869.

[18] A. Ferrari, P. R. Sala, A. Fasso, J. Ranft, FLUKA: A multi-particle transport code (Program version 2005), CERN-2005-10 (2005), INFN/TC $05 / 11$, SLAC-R-773CERN-2005-010.

[19] D. Newton, J. Knapp, A. Watson, The optimum distance at which to determine the size of a giant air shower, Astropart. Phys. 26 (2007) 414 arXiv:astro-ph/0608118.

[20] D. Maurel, M. Ave, M. Roth, J. Gonzalez, Universality of the time structure of ground particle distributions and its application to the reconstruction of extensive air showers, Proc. of 33rd Int. Cosmic Ray Conf., Rio de Janeiro, Brazil, icrc2013-0600.pdf.

[21] X. Bertou, et al., Calibration of the surface array of the Pierre Auger Observatory, Nucl. Instrum. Meth. A568 (2006) 839.

[22] P. L. Ghia, et al., Testing the surface detector simulation for the Pierre Auger Observatory, Proc of 30th Int. Cosmic Ray Conf., Merida, Yucatan, Mexico, Vol. 4, 315, arXiv:0706.1212.

[23] H. Kawai, et al., Telescope array experiment, Nucl. Phys. Proc. Suppl. 175-176 (2008) 221. 
[24] V. Verzi, et al., The Pierre Auger Observatory: Contributions to the 33rd International Cosmic Ray Conference (ICRC 2013), Proc of 33rd Int. Cosmic Ray Conf., Rio de Janeiro, Brazil, in press, arXiv:1307.5059.

[25] T. Abu-Zayyad, R. Aida, M. Allen, R. Anderson, R. Azuma, et al., The Cosmic Ray Energy Spectrum Observed with the Surface Detector of the Telescope Array Experiment, Astrophys. J. 768 (2013) L1, arXiv:1205. 5067.

[26] R. Pesce, et al., The Pierre Auger Observatory I: The Cosmic Ray Energy Spectrum and Related Measurements, Proc. of 32nd Int. Cosmic Ray Conf., Beijing, China, Vol. 2, 214, arXiv:1107.4809.

[27] D. Ravignani, et al., The Pierre Auger Observatory: Contributions to the 33rd International Cosmic Ray Conference (ICRC 2013), Proc. of 33rd Int. Cosmic Ray Conf., Rio de Janeiro, Brazil, in press, arXiv:1307.5059. 